\title{
Uso de percepción remota y SIG en la selección de sitios de muestreo de suelos para la Región de Magallanes
}

\author{
Use of remote sensing and GIS in the selection of soil sampling sites \\ for the Magallanes Region
}

Muñoz, R. ${ }^{a}$, Radic S. $^{a *}$

${ }^{a}$ Universidad de Magallanes, Departamento de Ciencias Agropecuarias y Acuícolas, Punta Arenas, Chile.

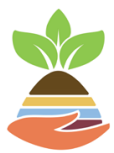

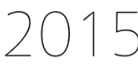

International

Year of Soils
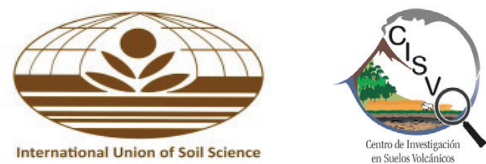

\section{A R T I CLE INF O}

Article history:

Received 18.08.2015

Accepted 17.03.2016

Keywords:

Open source

Vegetal communities

Number of required soil

sub-samples

Satellite images

Original Research Article,

Special Edition: International Year

of Soils (IYS)

Soil Science

*Corresponding author:

Sergio Radic

E-mail address:

sergio.radic@umag.cl

\section{A B S T R A C T}

The agricultural farms in Magallanes Region comprise vast areas, which makes complex the quantification of vegetal communities in all paddocks, and the planning of soil sampling based on these communities, due to the time and cost involved. This study proposes a method to determine homogenous zones for soil sampling, according to Geographic Information Systems and remote sensing techniques, based on open source data and software. The specific aim is determining the number of sub-samples to generate a soil composite sample. The results obtained showed a classification of vegetation with an $85.6 \%$ of global reliability from Landsat 8 data and identified the plane lands (50.3\% of farm total area) using Models of Digital Elevation of ASTER GDEM. The overall information allowed to generate 237.9 hectares defined like homogenous areas for different vegetal communities. Once the homogenous areas are determined, the sampling should be selected according to the community that will be assessed, and to the site that is intended to be studied or intervened. Subsequently, the estimation of the number of soil sub-samples is determined according to the area to sample and its homogeneity features. This procedure would help reduce mistakes in soil sampling.

\section{RESUMEN}

Los predios de uso agropecuario de la Región de Magallanes son de grandes superficies, por lo cual, es complejo cuantificar las comunidades vegetales que poseen, como también planificar muestreos de suelo en base a la distribución de sus comunidades, ya que implica tiempo y costos asociados. El presente estudio propone un método para la determinación de zonas homogéneas para muestreo de suelo, mediante técnicas de Sistemas de Información Geográfica (SIG) y percepción remota, basado en el uso de datos y softwares de libre disposición. También se propone las pautas para la determinación de la cantidad de sub-muestras, para generar una muestra compuesta de suelo. Los resultados permitieron obtener una clasificación de la vegetación con un $85,6 \%$ de fiabilidad global a partir de datos Landsat 8 e identificar los terrenos definidos como planos (50,3\% de la superficie total del predio) usando Modelos Digitales de Elevación (MDE) de ASTER GDEM. La integración de esta información, permitió obtener 237,9 hectáreas definidas como áreas homogéneas para las distintas comunidades vegetales. Una vez definidas las áreas homogéneas, el muestreo debería ser selectivo de acuerdo a la comunidad a evaluar y el sitio que se quiere diagnosticar o intervenir. Posteriormente, se realiza la estimación del número de sub-muestras de suelo según la superficie de hectáreas a muestrear y que posea las características de homogeneidad. Este procedimiento ayudaría a disminuir los errores producto del muestreo.

Palabras clave: Código abierto, comunidades vegetales, número requerido de sub-muestras de suelo, imágenes de satélite.

\section{INTRODUCCIÓN}

En la Región de Magallanes existen cerca de 13 millones de hectáreas (ha), de las cuales según el Cen- so Agropecuario (Instituto Nacional de Estadísticas, 2007), 5.357.442 hectáreas tienen relación con predios de uso agropecuario, los que poseen una superficie promedio aproximada de 4000 hectáreas. Con una 
producción ganadera principalmente ovina, basada en su mayoría en sistemas de producción extensivos con pastoreo de praderas naturales. Para la región de Magallanes, el Servicio Agrícola y Ganadero - SAG (2003, 2004a, 2004b) realizó un estudio de la vegetación que abarco alrededor de 5 millones de hectáreas de uso agropecuario, donde se determinan al menos siete comunidades vegetales importantes; bosque, coironal, murtilla, turba, mata, vegas y praderas, las cuales presentaban una superficie aproximada de 1,$2 ; 1,1 ; 0,8$; 0,4; 0,3; 0,2 y 0,2 millones de hectáreas respectivamente. Todos los predios de la región poseen una o varias de estas comunidades, por lo cual, es importante poder determinar la superficie que abarcan en cada unidad.

Posterior al año 2000 hasta la fecha, existe un aumento en la superficie de praderas sembradas y mejoradas en Magallanes, producto de una iniciativa público-privada (programa de recuperación de suelos degradados del Servicio Agrícola y Ganadero). Este aumento ha generado la necesidad de evaluar la condición de los suelos previo a la siembra. En un programa de análisis de suelos es relevante la obtención de una muestra que sea representativa del sitio, producto que las muestras mal tomadas son generalmente los errores más significativos, los cuales no se pueden corregir en las fases subsecuentes. Una muestra mal tomada puede causar errores en el análisis y posterior diagnóstico de la fertilidad de un suelo desde un 50\% (Bernier, 2000) hasta un 85 \% (Domínguez, 1997).

La gran superficie de los predios agropecuarios de la Región de Magallanes, generan la necesidad de conocer con exactitud el tamaño de los potreros y la superficie de las comunidades vegetales presentes. Estas comunidades pueden presentar diferentes características de suelo (Collantes et al., 1999; Radic et al. 2013; Ivelic-Sáez et al. 2014), por lo cual, es necesario obtener muestras de suelo para evaluación de la fertilidad según las comunidades vegetales existentes.

El uso de métodos convencionales para la cuantificación de las comunidades vegetales a nivel predial y la selección de áreas representativas para posteriores muestreos, resulta complejo y con un costo elevado. Los Sistemas de Información Geográfica (SIG) y las tecnologías asociadas como la Percepción Remota (PR) y los Sistemas de Posicionamiento Global (GPS), tienen un rol cada vez más importante en la agricultura, con diversas aplicaciones en escalas globales, regionales y locales, donde se pueden destacar aquellas relacionadas con la agricultura de precisión (Wilson, 1999). En este escenario, varios autores señalan la incorporación de técnicas de SIG y PR con el muestreo de suelos (Mallarino y Wittry, 2004; Dinkins y Jones, 2008; Peng et al., 2013), permitiendo una descripción más precisa de los patrones espaciales del territorio y la ubicación de cada una de las muestras tomadas.

En la actualidad, las políticas de diversas agencias espaciales permiten que exista una masificación de los datos satelitales disponibles, asegurando una continuidad, asequibilidad y accesibilidad de los datos (Turner et al., 2014). A su vez el incremento en la última década de programas libres y de código abierto (Open Source), permiten almacenar, editar, analizar y visualizar información geográfica de diversa índole (Steiniger y Hunter, 2012), generando un escenario ideal para incorporar estas tecnologías para mejorar las técnicas de selección de zonas para el muestreo de suelos en diferentes lugares y diversas escalas.

El presente estudio propone un método para la determinación de zonas homogéneas para muestreo de suelo, mediante técnicas de Sistemas de Información Geográfica y Percepción Remota, basado en el uso de datos y programas de libre disposición. También propone las pautas para la determinación del número de sub-muestras de suelo para generar una muestra compuesta en sistemas de praderas extensivas.

\section{MATERIALES Y MÉTODOS}

Se propone una metodología para la determinación de unidades homogéneas de muestreo a partir de la integración cartográfica de comunidades vegetales y características topográficas del terreno (pendiente), generada a partir de datos satelitales. También se propone pautas para la determinación de la cantidad de sub muestras, para generar una muestra compuesta de suelo. El método, se basa en la utilización de datos y softwares de libre disposición (Open Source). La metodología se puede resumir en cuatro pasos: 1 . Área de estudio; 2. Obtención de los datos; 3. Procesamiento; 4. Selección de las unidades de muestreo y determinación de numero de sub-muestras.

\section{Área de estudio.}

El área de estudio corresponde a una estancia, ubicada en la provincia de Tierra del Fuego, al sur de la región de Magallanes. El predio es de uso ganadero y posee una superficie de 4120 ha. La selección del predio se fundamentó en la variabilidad de las comunidades vegetales y características topográficas que presentaba, para de esta manera realizar el análisis en un predio de alta complejidad.

Obtención de los datos. Los datos utilizados corresponden a imágenes satelitales multiespectrales y a modelos digitales de elevación (MDE) obtenidos a partir de sensores remotos. Las imágenes satelitales utilizadas fueron obtenidas por el satélite Landsat 8 (cuadro 1). Los datos Landsat han sido utilizados en el monitoreo de coberturas terrestres desde los años '70, inicios de dicho programa satelital de la NASA (National Aeronautics and Space Administration) (Roy et al., 2014). En la actualidad, estas imágenes son las que poseen la mayor 

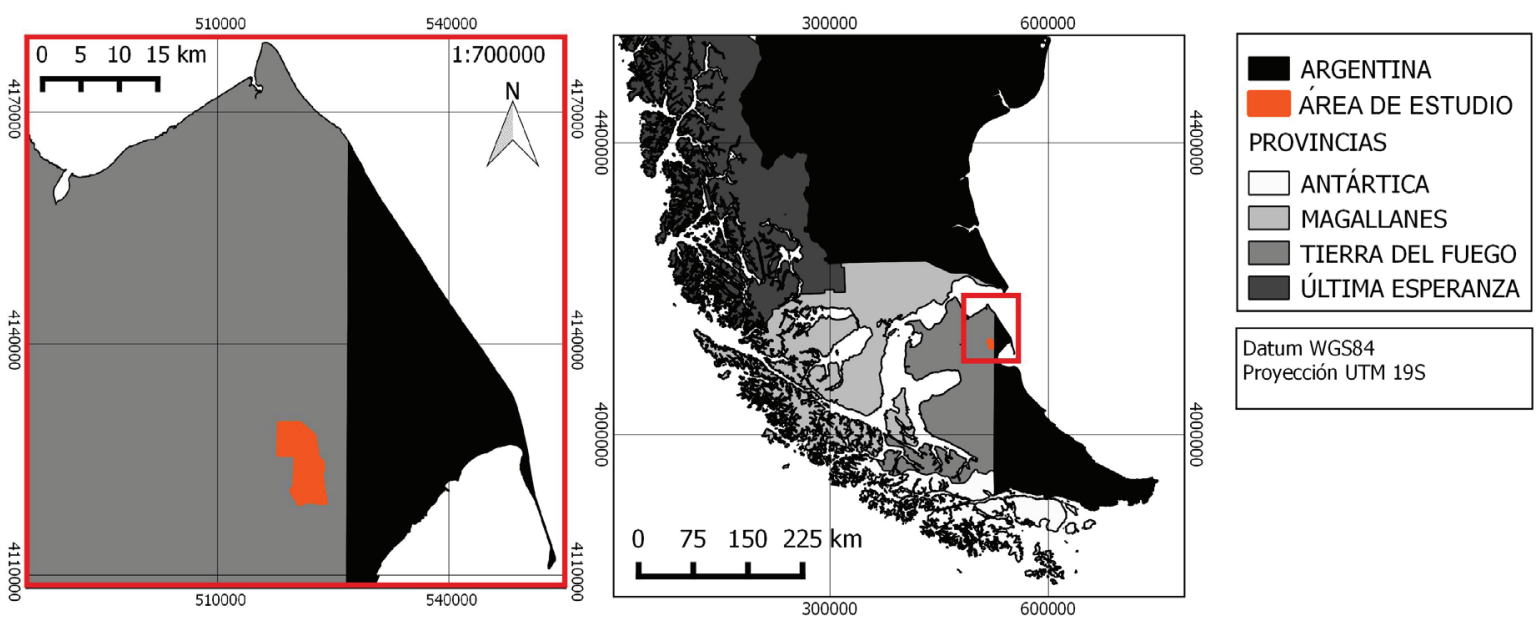

Figura 1. Área de estudio.

Figure 1. Study area.

Cuadro 1. Bandas individuales para Landsat 8, características espectrales (longitud de onda central y ancho de banda) y resolución espacial.

Table 1. Individual bands for Landsat 8, spectral features (central wavelength and bandwidth) and spatial resolution.

\begin{tabular}{lccc}
\hline Nombre de banda & $\begin{array}{c}\text { Longitud de onda central } \\
(\mathrm{nm})\end{array}$ & $\begin{array}{c}\text { Ancho de banda } \\
(\mathrm{nm})\end{array}$ & $\begin{array}{c}\text { Resolución espacial } \\
(\mathrm{m})\end{array}$ \\
\hline Coastal/Aerosol & 443 & 20 & 30 \\
Blue & 482 & 65 & 30 \\
Green & 562 & 75 & 30 \\
Red & 655 & 50 & 30 \\
NIR (Near Infrared) & 865 & 40 & 30 \\
SWIR1 (Short Wave Infrared) & 1610 & 100 & 30 \\
SWIR2 & 2200 & 200 & 30 \\
Panchromatic & 590 & 180 & 15 \\
Cirrus & 1375 & 30 & 30 \\
TIR1 & 10900 & 570 & $100(30) *$ \\
TIR2 & 12000 & 1000 & $100(30) *$ \\
\hline
\end{tabular}

Fuente: Knight y Kvaran (2014) y Reuter et al. (2015).

*Las bandas TIRS son obtenidas con 100 metros de resolución espacial, pero son remuestreadas a 30 metros.

resolución espacial, dentro de las imágenes de libre disposición y que tienen una cobertura a escala global (Johnson et al., 2014). El satélite Landsat 8 lleva abordo 2 sensores, OLI (Operational Land Imager) y TIRS (Thermal Infrared Sensor). La resolución temporal de estas imágenes es de 16 días y pueden ser descargadas a través de los sitios web http://earthexplorer.usgs.gov/ y http://glovis.usgs.gov/. La imagen descargada (path = 227, row $=97$ ) corresponde a una escena adquirida por dicho sensor el 07 de marzo del 2015 a las 14:01 hr. Esta imagen fue seleccionada ya que no presentaba presencia de nubes en el área de estudio y no correspondía a la época de invierno. La imagen posee un nivel de procesamiento "level 1T - Terrain correctión".

Un Modelo Digital de Elevación (MDE) puede ser definido como una representación matricial regular de la variación continua del relieve en el espacio $(\mathrm{Bu}-$ rrough, 1986). Estos MDE y sus parámetros derivados como la pendiente, la exposición, entre otros, constituyen una importante herramienta para el análisis digital del terreno (Athmania y Achour, 2014). Uno de los MDE más populares en los últimos años corresponde a 
ASTER GDEM (Global Digital Elevation Model), el cual es generado a partir de técnicas de fotogrametría usando imágenes estereoscópicas (pares) obtenidas a partir del sensor ASTER (Advanced Spaceborne Thermal Emission and Reflection Radiometer). Este producto se encuentra en su segunda versión, poseen 30 metros de resolución espacial y se encuentra disponible en el sitio web http://gdem.ersdac.jspacesystems.or.jp/. Cabe destacar que también existen otras alternativas como el MDE de SRTM (Shuttle Radar Topography Mission) con un resolución espacial de $30 \mathrm{~m}$ (de libre disposición) y datos comerciales como el TanDEM X y los generados a partir de tecnología LIDAR.

Procesamiento de los datos. En el caso de los datos satelitales Landsat, el procesamiento se orienta a generar una clasificación digital de las comunidades vegetales presentes en el sitio de interés, mientras que para el MDE el proceso está enfocado en obtener una imagen de pendiente del terreno categorizada en plano $(0-10 \%)$ y ondulado (11-30\%) (SAG, 2003). Para la extracción de las comunidades vegetales a partir de una imagen es necesario seguir tres pasos fundamentales: Pre procesamiento de los datos, clasificación y validación. Antes de ser utilizadas e interpretadas, las imágenes deben pasar por algunos procesos que deben ser realizados para darle un sentido físico a los valores y corregir ciertos errores que se producen que son inherentes al sistema de adquisición. La energía captada por el sensor se almacena como niveles digitales (ND). La conversión de los ND a variables físicas es un paso importante en la fase de interpretación de las imágenes, ya que permite trabajar con variables físicas de significado estándar comparables con un mismo sensor a lo largo del tiempo y entre distintos sensores (Chuvieco, 2006). Por lo anterior, es que las imágenes deben ser calibradas y convertidas a reflectancia. Cabe mencionar que a partir del año 2015, se encuentra disponible un nuevo producto de Landsat 8, denominado "Surface Reflectance", el cual ya incorpora el proceso de conversión antes mencionado.

Por otra parte, la cartografía temática (comunidades vegetales en este caso) a partir de datos generados por teledetección, generalmente se basan en la clasificación de la imagen (Foody, 2005), proceso extensamente estudiado (Maulik y Chakraborty, 2013; Phillips et al., 2014). Una manera de extraer información temática de datos satelitales es a través de la interpretación visual, sin embargo, esta se limita a la experticia del operador y a una subutilización de toda la información disponible en las imágenes, es por esto que la clasificación automática es más adecuada, sobre todo en extensas áreas (Rozenstein y Karnieli, 2011).

Existen dos pasos generales en una clasificación supervisada, la que a partir de un conjunto de píxeles conocidos, intenta asignar las clases a aquellos conjuntos desconocidos (Chuvieco, 2006), estas corresponden a la selección de áreas de entrenamiento y a la aplicación de algún algoritmo de asignación de los pixeles a una clase, de acuerdo a las estadísticas de dichas áreas. Finalmente es necesario evaluar la precisión de la clasificación, para lo cual, se tabulan los datos a través de una matriz de confusión, donde se derivarán índices de concordancia, como el de fiabilidad global. En el presente caso de estudio, se utilizaron regiones de entrenamiento obtenidas a partir de terreno, de las cuales el $70 \%$ se usaron para el proceso de asignación, mientras que el resto se emplearon en la validación. Para la asignación se utilizó el algoritmo de máxima verosimilitud, uno de las más utilizados en teledetección de datos provenientes de sensores ópticos (Yonezawa, 2007; Miao et al., 2011). Este clasificador asume que los datos siguen una distribución normal, de esta manera se puede describir una clase a partir de una función de probabilidad y así poder determinar la probabilidad de que un pixel pertenezca a esta clase. Según Richards (1999) la función que utiliza esta dada por:

$g_{i}(x)=\ln p\left(\omega_{i}\right)-\frac{1}{2} * \ln \left[\sum_{i}\right]-\frac{1}{2} *\left(x-m_{i}\right)^{t} \sum_{i}^{-1}\left(x-m_{i}\right)$

Dónde:

$\mathrm{i}=$ Clase.

$\mathrm{x}=$ Numero de bandas.

$p\left(\omega_{i}\right)=$ Probabilidad de que clase $\omega_{i}$ ocurra en la imagen.

$\left|\Sigma_{i}\right|=$ Determinante de la matriz de covarianza en los datos de la clase $\omega_{\mathrm{i}}$.

$\Sigma_{i}^{-1}=$ Matriz inversa.

$\mathrm{m}_{\mathrm{i}}=$ Vector promedio.

Para realizar estos procesos se utilizó el software libre y de código abierto QGIS v 2.8.1 (Quantum GIS Development Team, 2015), el cual incorpora un complemento denominado Semi-Automatic Classification Plugin (Congedo y Munafò, 2012), que provee herramientas para ejecutar de manera semi-automática todos los procesos antes mencionados.

Desde un MDE es posible derivar una serie de parámetros que son de gran utilidad para caracterizar el territorio desde el punto de vista topográfico, entre los que podemos mencionar la pendiente, la exposición de las laderas, las sombras, entre otros. La pendiente puede ser definida como aquel ángulo que está formado entre el vector normal sobre el terreno en un punto determinado y su vertical. El formato raster que poseen los MDE permite realizar estimaciones por vecindad, la cual es utilizada en un entorno SIG para el cálculo de parámetros topográficos como la pendiente. El software QGIS v2.8.1 incorpora un complemento denominado Raster Based Terrain Analysis, el cual permite extraer la pendiente, entre otros parámetros, a partir de un MDE. Esta herramienta utiliza derivada parcial de primer orden sobre un kernel (ventana móvil) de 3x3 píxeles, asignando al valor 
central de dicho kernel el cálculo obtenido a partir de los valores de sus 8 píxeles vecinos (Burrough et al., 1998)

Selección de las unidades de muestreo y determinación del número de sub-muestras. Una vez generada la clasificación de las comunidades vegetales y la imagen de pendiente del terreno, es posible utilizar ambos datos para obtener unidades homogéneas de muestreo que cumplan con las siguientes condiciones: (1) pertenezcan a una clase homogénea de comunidad vegetal en particular (de acuerdo a la clasificación de vegetación obtenida), (2) que se encuentren en un terreno con pendiente plano $(0-10 \%)$ y (3) que los polígonos que cumplan estas dos condiciones anteriores, tengan una superficie mínima de tres hectáreas. Lo anterior se basa, en que algunos autores (Mallarino y Wittry, 2004; Thompson et al., 2004) señalan que existe una disminución de la variabilidad cuando se integran diferentes capas de información para seleccionar las zonas de muestreo. Para esto se siguieron los siguientes pasos: (1) convertir la clasificación de vegetación y la imagen de pendiente desde formato raster a vectorial, (2) crear una capa que divida toda el área de estudio en celdas idénticas de 5 ha cada una, en donde las áreas homogéneas, enmarcadas dentro de cada celda, correspondan a superficies mayores a 3 ha, (3) unir toda la información mediante el geoproceso de "unión" y (4) agregar la superficie de cada polígono a la tabla de atributos.
Con toda la información contenida en la capa de unión, es posible generar una consulta espacial que permita seleccionar las áreas que cumplan con las condiciones anteriormente mencionadas.

Una vez seleccionadas las unidades de muestreo, se determina la superficie y la cantidad de sub-muestras a obtener para generar la muestra compuesta de suelo, considerando como base 12 sub-muestras por hectárea de características homogéneas. Producto de la gran variabilidad en el número de sub-muestras señaladas por diversos autores (cuadro 2), los cuales en su mayoría realizan las referencias de acuerdo a la experiencia o a citas de otros trabajos, se utilizó la media de los valores encontrados. Lopetegui (2000), fue uno de los únicos trabajos indicados en el cuadro 2, que realizó un estudio para estimar el número de sub-muestras para conformar una muestra compuesta según el coeficiente de variación (CV) con el cual se desea trabajar. Sus resultados indicaron que entre 1 y 12 sub-muestras son requeridas para conformar una muestra compuesta utilizando un CV del $10 \%$, para los siguientes parámetros del suelo: $\mathrm{pH}$ al agua, $\mathrm{pH} \mathrm{CaCl}{ }_{2}$, materia orgánica, calcio, sodio, suma de bases, fósforo, potasio, aluminio intercambiable y magnesio, ordenados de menor a mayor número de sub-muestras. Según lo anterior, con 12 sub-muestras como mínimo sería suficiente para conformar una muestra compuesta utilizando un CV del 10\%, misma cantidad obtenida de la media de los trabajos señalados en el cuadro 2.

Cuadro 2. Número de sub-muestras de suelo a extraer por superficie homogénea de muestreo, según diferentes autores.

Table 2. Number of soil sub-samples to be extracted per homogeneous sampling area, according to different authors.

\begin{tabular}{|c|c|c|c|}
\hline & & MUE & OMPUESTA \\
\hline & $\begin{array}{c}\text { Superficie homogénea } \\
\text { en hectáreas }\end{array}$ & $\begin{array}{c}\text { Número de } \\
\text { sub - muestras }\end{array}$ & $\begin{array}{c}\text { Sub-muestras en relación a } 1 \\
\text { hectárea* }\end{array}$ \\
\hline FAO, 1986 & Por unidad de muestreo & 15 a 20 & 17 \\
\hline Finck, 1988 & 1 & 20 a 40 & 40 \\
\hline Rodríguez, 1991 & Por unidad de muestreo & 10 & 10 \\
\hline CFASIC, 1995 & Por unidad de muestreo & 10 a 20 & 15 \\
\hline Gerding y Schlatter, 1996 & Por unidad de muestreo & 3 a 10 & 7 \\
\hline Domínguez, 1997 & 1 & 20 a 30 & 30 \\
\hline Guerrero, 2000 & 10 & 15 a 40 & 4 \\
\hline Bernier, 2000 & 10 & 20 a 25 & 3 \\
\hline Lopetegui, 2000 & 1 & 1 a 12 & 12 \\
\hline NPFI, 2001 & 4 & 12 a 20 & 5 \\
\hline Tejeda et al., 2001 & 10 & 10 & 1 \\
\hline ERC, 2004 & 5 & 20 & 4 \\
\hline Matus, 2006 & 10 & 20 a 30 & 3 \\
\hline Brady y Weil, 2008 & 10 & 12 a 15 & 2 \\
\hline Frame y Laidlaw, 2011 & Por unidad de muestreo & 20 a 30 & 25 \\
\hline MEDIA & & & 12 \\
\hline
\end{tabular}

*Cifras aproximadas al entero. Cuando se presentó un rango de sub-muestras se optó por el valor mayor cuando la superficie señalada era numérica, en los casos en que se menciona "por unidad de muestreo" se ocupó la media del rango. 


\section{RESULTADOS Y DISCUSIÓN}

La validación de la clasificación obtenida a partir de la imagen Landsat 8 utilizada (figura 2), arrojo una fiabilidad global de $85,6 \%$ (píxeles correctamente clasificados respecto del total de píxeles verificados), lo que puede considerarse como una buena clasificación. De acuerdo a la matriz de confusión la clase praderas sembradas, presenta un porcentaje de $22,41 \%$ de píxeles asignados erróneamente a la clase Vegas. Coherentemente con lo anterior, la clase praderas sembradas presenta un 38,24\% de error de comisión (cuadro 3).

Diversos autores afirman que es posible aumentar esta precisión con la incorporación de series de tiempo de imágenes Landsat 8 a la clasificación (Senfetal.,2014; Knudby et al., 2014). Las clases más representativas del predio corresponden a comunidades de matorrales de
Chiliotrichum diffusum (G.Forst) Kuntze., coironales de Festuca gracillima Rothm. y vegas, con una ocupación de 35, 25 y $13 \%$ respectivamente del total del predio, mientras que las clases praderas sembradas o pastos cortos y comunidades de murtillares de Empetrum rubrum Vahl ex Willd. solo representaron el 7 y $9 \%$ respectivamente (cuadro 4). Leon et al. (1998) describe la vegetación de esta zona (entre los paralelos $52^{\circ}$ y $54^{\circ} \mathrm{S}$ ), destacando la frecuencia de estepa graminosa dominada por Festuca gracillima, de Matorrales de Chilliotrichium diffusum y Murtillares de Empetrum rubrum. Los resultados de la clasificación obtenida son coherentes con esta descripción a excepción de los murtillares que presentaron una baja ocupación dentro del predio (cuadro 4).

En cuanto a la clasificación de la imagen de pendiente en porcentaje, el 50,3\% de la superficie total del

Cuadro 3. Izquierda: Matriz de confusión para la clasificación obtenida, los valores se muestran en porcentaje de píxeles; Derecha: Errores de comisión y omisión en porcentaje.

Table 3. Left: Confusion matrix for the obtained classification, the values are expressed in pixels percentage; Right: commission and omission errors are expressed in percentage.

\begin{tabular}{lrrrrrrrrrrr}
\hline & \multicolumn{1}{c}{} & \multicolumn{1}{c}{ \% de píxeles } & \multicolumn{4}{c}{ Error (\%) } \\
\hline & $\mathbf{1}$ & $\mathbf{2}$ & $\mathbf{3}$ & $\mathbf{4}$ & $\mathbf{5}$ & $\mathbf{6}$ & $\mathbf{7}$ & Total & Comisión & Omisión \\
\hline Murtillar (1) & 96,30 & 0,00 & 0,00 & 0,00 & 0,00 & 21,74 & 31,09 & 54,09 & 17,24 & 3,70 \\
Cuerpo de agua (2) & 0,00 & 87,04 & 0,00 & 0,00 & 0,00 & 0,00 & 0,00 & 6,74 & 0,00 & 12,96 \\
Vega (3) & 0,00 & 3,70 & 77,59 & 4,55 & 8,70 & 0,00 & 0,00 & 7,17 & 10,00 & 22,41 \\
Praderas sembradas (4) & 0,00 & 0,00 & 22,41 & 95,45 & 0,00 & 0,00 & 0,00 & 4,88 & 38,24 & 4,55 \\
Coironal (5) & 0,00 & 9,26 & 0,00 & 0,00 & 91,30 & 0,00 & 0,00 & 3,73 & 19,23 & 8,70 \\
Suelo desnudo (6) & 0,93 & 0,00 & 0,00 & 0,00 & 0,00 & 78,26 & 0,00 & 3,01 & 14,29 & 21,74 \\
Matorral (7) & 2,78 & 0,00 & 0,00 & 0,00 & 0,00 & 0,00 & 68,91 & 20,37 & 6,34 & 31,09 \\
\hline Total & 100 & 100 & 100 & 100 & 100 & 100 & 100 & 100 & & \\
\hline
\end{tabular}

Cuadro 4. Superficie clasificada según cobertura, indicando número de pixeles, porcentaje del total y área en hectáreas.

Table 4. Classified area according to cover, indicating pixels number, total percentage and area in hectares.

\begin{tabular}{lccc}
\hline Clase & $\mathbf{N}^{\circ}$ píxeles & Porcentaje (\%) & Área (ha) \\
\hline Murtillar & 4100 & 9 & 369 \\
Cuerpos de agua & 692 & 2 & 62 \\
Vegas & 5821 & 13 & 524 \\
Praderas sembradas & 3159 & 7 & 284 \\
Coironal & 10937 & 25 & 984 \\
Suelo desnudo & 4222 & 9 & 380 \\
Matorral & 15576 & 35 & 1.402 \\
\hline
\end{tabular}


Combinación de bandas R:G:B - 5:4:3

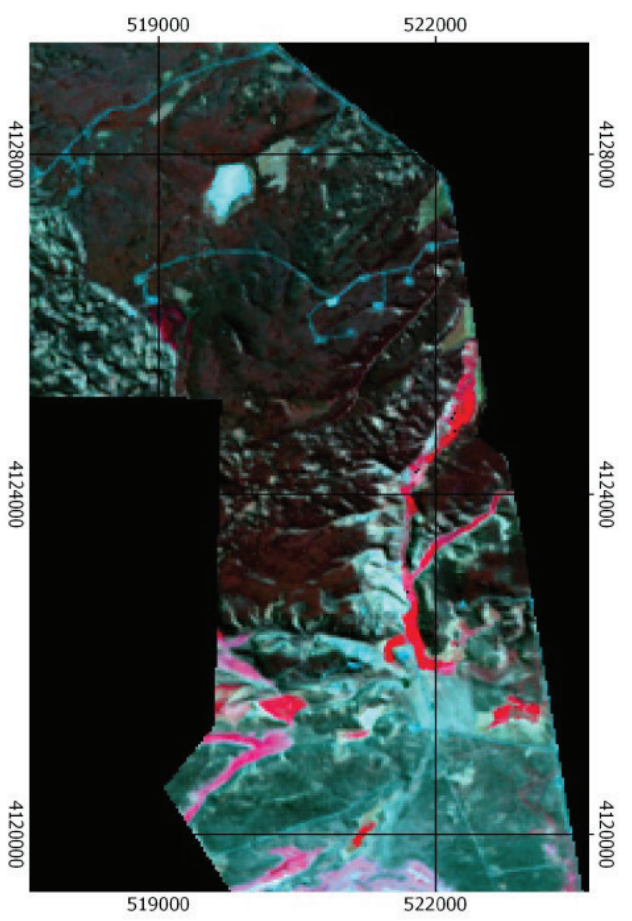

Rangos de pendiente (\%).

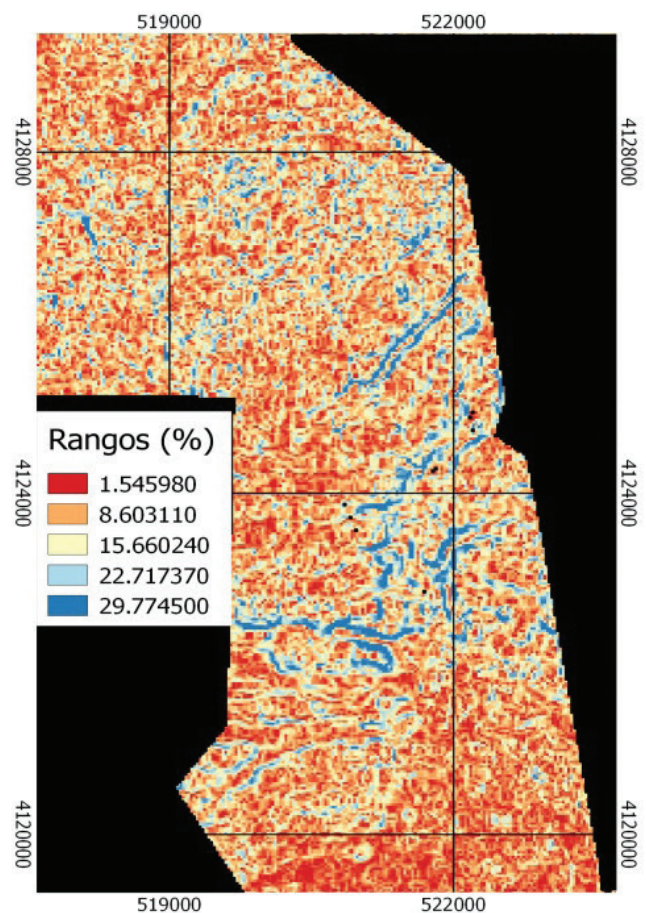

Imagen clasificada.

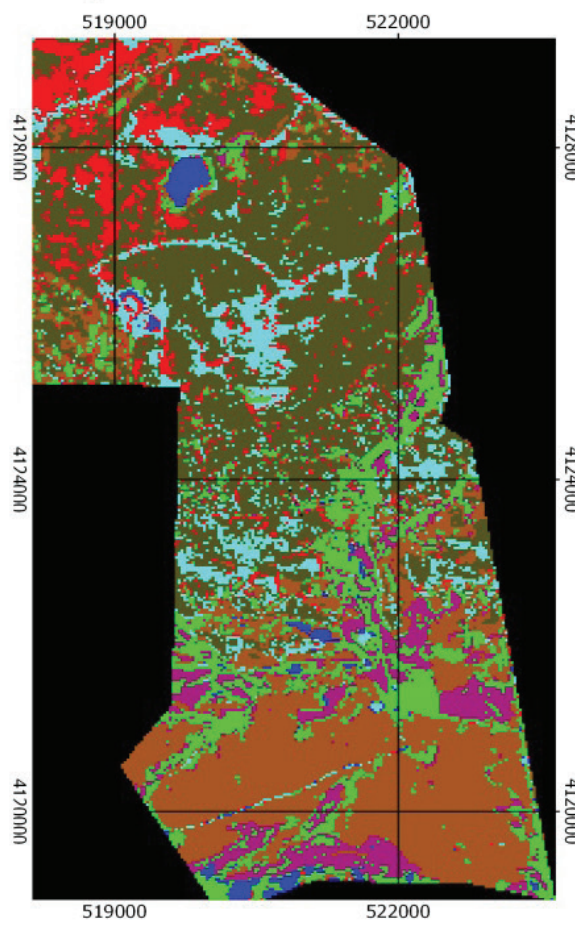

\begin{tabular}{|l|}
\hline 1 - Murtillar \\
2 - Cuerpo de agua \\
3 - Vega \\
4 - Praderas sembradas \\
5 - Coironal \\
6 - Suelo desnudo \\
7 - Matorral \\
\hline
\end{tabular}

莒

楞

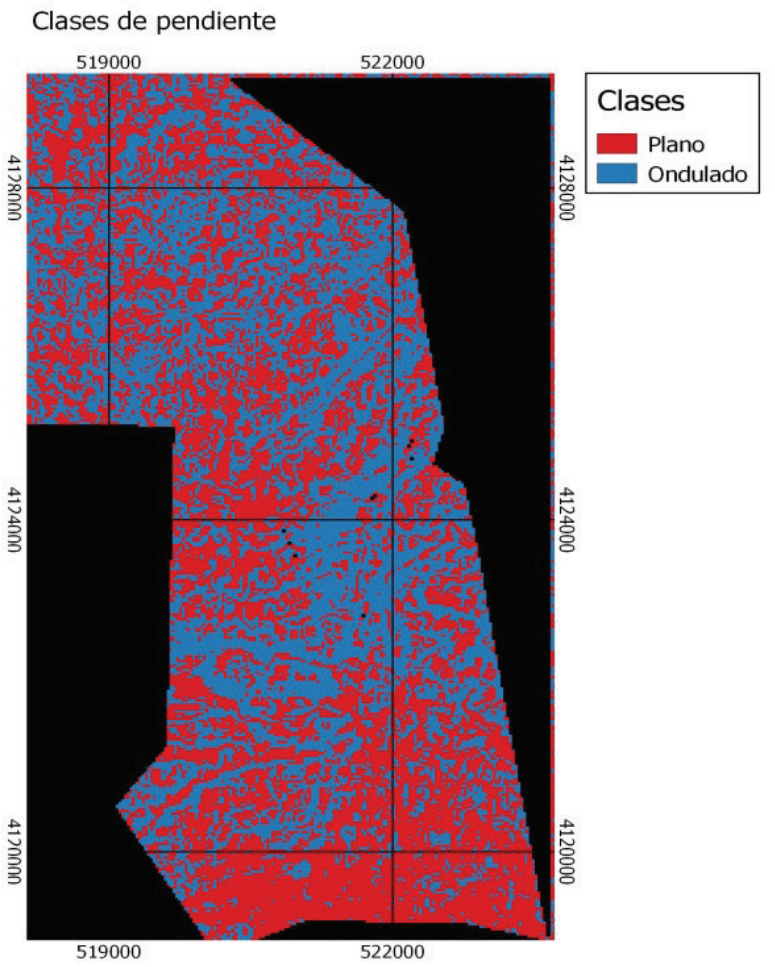

Figura 2. Arriba izquierda: imagen landsat 8 del predio, en combinación de bandas R:G:B - 5:4:3; Arriba derecha: imagen clasificada para el predio; Abajo izquierda: Rangos de pendiente a partir de ASTER GDEM; Abajo derecha: Pendiente reclasificada en categorías plano (0 - 10\%) y ondulado (11 - 35\%).

Figure 2. Above left: farm landsat 8 image, bands combination R:G:B - 5:4:3; above right: classified image for a farm; bottom left: slope range from ASTER GDEM; bottom right: reclassified slope in categories plain (0 - 10\%) and undulating (11 - 35\%). 
predio, se categorizó en la clase plano, mientras que solo el $49,7 \%$ restante fue asignado a la clase ondulado. La fiabilidad de estos resultados, se encuentra directamente relacionada con la calidad del MDE. La precisión de ASTER GDEM ha sido evaluada por diversos autores, Athmania y Achour (2014) encontraron un error medio cuadrático de 5,3 m, comparando con datos tomados por GPS, no obstante otros autores afirman que se podrían obtener mejores resultados, al generalizar ASTER GDEM pasando de una resolución espacial (tamaño de píxel) de 30 a 90 o 100 m (Hengl y Reutter, 2011).

Las áreas homogéneas obtenidas a partir de la integración de la clasificación de coberturas terrestres y la clasificación de pendientes (Figura 3), corresponden a 3,$06 ; 6,55 ; 3,6 ; 202 ;$ y 22,6 hectáreas para la clase murtillar, vegas, praderas sembradas, coironal y matorrales respectivamente. A pesar que la clase matorrales ocupó mayor superficie dentro del predio, fue la clase coironal la de mayor superficie definida como áreas homo- géneas, debido a que la clase matorral se encontraba principalmente en los sectores ondulados.

Según lo señalado en el cuadro 2, la cantidad mínima de sub-muestras a extraer por hectárea serían 12, pero existen situaciones habituales en la región donde se planifica sembrar o regenerar sectores planos y homogéneos que pueden llegar hasta 30 o más hectáreas. En este contexto, la cantidad de sub-muestras a obtener podrían ser 360 (12 sub-muestras por 30 hectáreas), lo cual genera una pérdida de tiempo y dificulta la logística en el trabajo. Por lo anterior, se plantea la posibilidad de disminuir el número de sub-muestras por hectárea a medida que aumenta la superficie a muestrear, de tal manera de disminuir el número total de sub-muestras (cuadro 5). Considerando los supuestos de homogeneidad de la unidad de muestreo mencionados anteriormente y la utilización de imágenes satelitales para la determinación de los sitios. En relación a la idea anterior, Thompson et al. (2004) señala que la
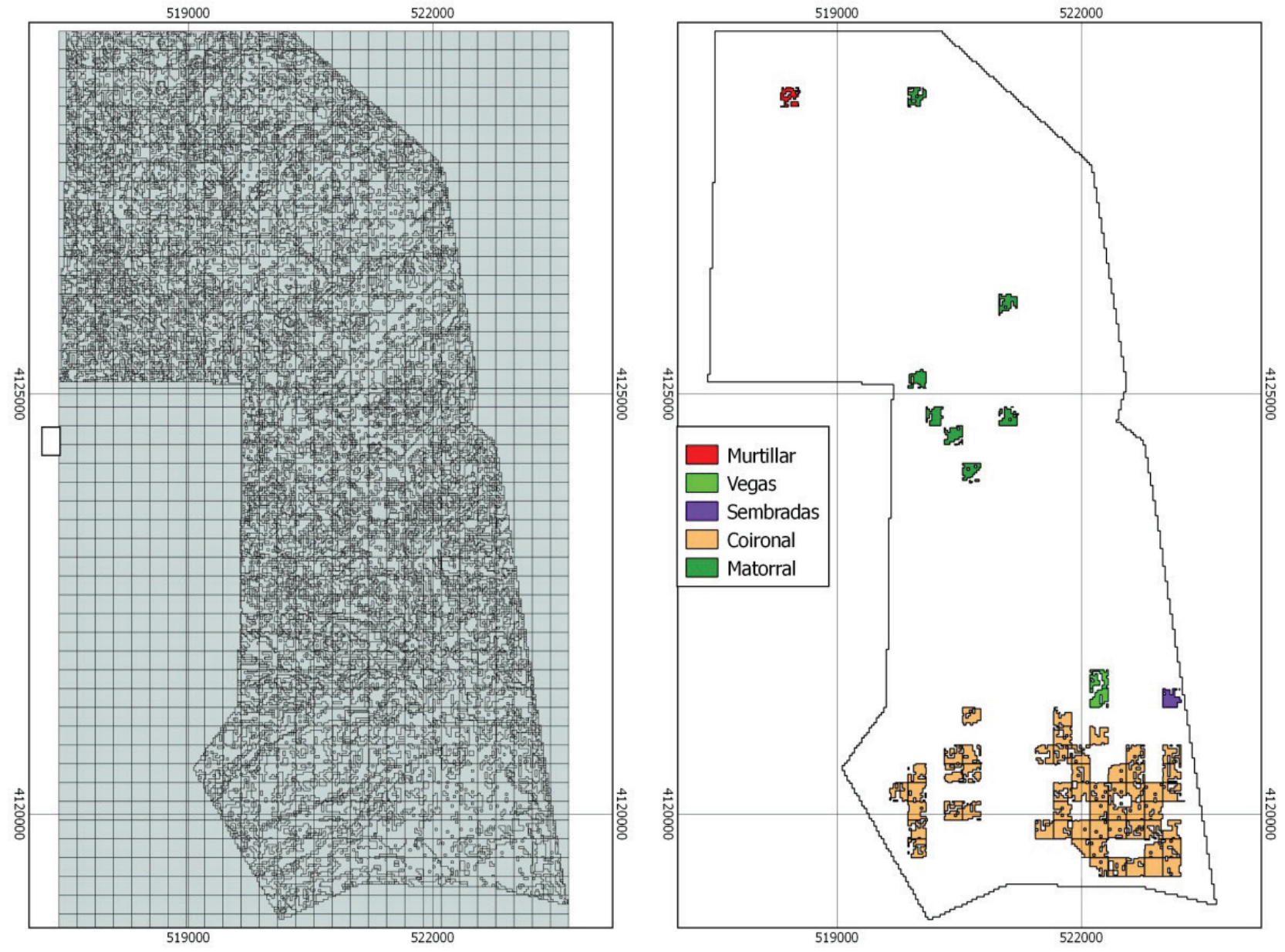

Figura 3. Izquierda: Capa vectorial que contiene la información de las clases vegetales, las clases de pendiente y las celdas de 5 hectáreas; Derecha: Áreas homogéneas identificadas según clases de vegetación.

Figure 3. Left: vector layer containing information about vegetal communities, the types of of slope and 5 hectares cells; Right: homogeneous areas identified according to vegetation classes. 
Cuadro 5. Número de sub-muestras de suelo a extraer según la superficie homogénea a muestrear, expresada en hectáreas (ha).

Table 5. Number of soil sub-samples to be extracted according to homogeneous area to sample, in hectares (ha).

\begin{tabular}{cccc}
\hline $\begin{array}{c}\text { Valor de cambio significati- } \\
\text { vo según Pearson* }\end{array}$ & $\begin{array}{c}\text { Superficie homogénea } \\
\text { a muestrear (ha) }\end{array}$ & $\begin{array}{c}\text { Número de sub-muestras mínima por } \\
\text { hectárea de superficie homogénea }\end{array}$ & $\begin{array}{c}\text { Número de sub-muestras } \\
\text { totales a extraer }\end{array}$ \\
\hline 0,997 & 1 & 12 & 12 \\
0,950 & 2 & 11 & 22 \\
0,878 & 3 & 11 & 33 \\
0,811 & 4 & 10 & 40 \\
0,754 & 5 & 9 & 45 \\
0,707 & 6 & 9 & 54 \\
0,632 & 8 & 8 & 64 \\
0,576 & 10 & 7 & 70 \\
0,449 & 15 & 5 & 75 \\
0,423 & 20 & 5 & 100 \\
0,349 & 30 & 4 & 120 \\
0,288 & 40 & 3 & 120 \\
0,273 & 50 & 3 & 150 \\
0,195 & 100 & 2 & 200 \\
\hline
\end{tabular}

* Valores obtenidos de la tabla de correlación de Pearson citada por Steel y Torrie (1980).

utilización de las imágenes satelitales en la selección de sitios de muestreo reducen el CV, mientras que FAO (1973), indica que la variación en el nivel de nutrientes dentro de una determinada superficie de suelo es casi la misma que dentro de una superficie mayor. Roberts y Henry (2001), señalan que se ha demostrado que el número de sub-muestras se incrementa muy poco a medida que se incrementa la superficie a muestrear, y que este incremento no es proporcional al incremento de la superficie.

El valor de cambio significativo según correlación de Pearson (cuadro 5), se obtuvo utilizando los valores significativos al 5\% señalados en la tabla. La columna que indica el número de sub-muestras mínima por hectárea de superficie homogénea, se obtuvo multiplicando el valor de cambio asociado a una superficie homogénea que se desee muestrear por el valor base de sub-muestras para una hectárea (12), redondeando el resultado al entero más próximo. Posteriormente se dividió por el valor de cambio de Pearson asociado a este valor base. La columna que indica número de submuestras totales a extraer, se obtuvo multiplicando la superficie homogénea a muestrear por el número de sub-muestras mínima por hectárea de superficie homogénea. De esta forma, la estimación para la obtención del número de sub-muestras de suelo estaría relacionada al valor de cambio indicado por Pearson, pudiendo reducir el número de sub-muestras totales a extraer para una determinada superficie a muestrear. Para el caso mencionado de un muestreo de suelo para 30 hectáreas, la cantidad de sub-muestras a colectar en esa superficie homogénea se reduciría a 120. Esta propuesta debería validarse en terreno con estudios posteriores para los diversos parámetros de suelo a evaluar, ya que algunos autores (Lopetegui, 2000; Roberts y Henry, 2001; Yu et al. 2011; Rodrigues et al. 2012) señalan que el número de muestras para determinar la distribución espacial de nutrientes depende del parámetro de suelo estudiado.

Según los resultados obtenidos en la clasificación vegetal realizada, se encontraron 15 áreas homogéneas de muestreo (cuadro 6) donde sería posible realizar intervenciones, dependiendo de la comunidad vegetal, como fertilización, siembra o regeneración, entre otros trabajos. En estas áreas, es necesario previamente un muestreo de suelo para conocer las características de los sitios, obteniendo el número de sub-muestras por unidad de muestreo según lo indicado en el cuadro 5. Esta propuesta para la obtención del número de submuestras de suelo para generar una muestra compuesta, ayudaría a disminuir el tiempo de trabajo y a facilitar la logística en terreno para las condiciones imperantes en la Región de Magallanes u otras regiones de similares características. 
Cuadro 6. Superficie de las comunidades homogéneas determinadas, indicando el número de sub-muestras de suelo en cada área.

Table 6. Area of determined homogeneous communities, indicating the number of soil sub-samples in each area.

\begin{tabular}{|c|c|c|}
\hline $\begin{array}{l}\text { Comunidad homogénea } \\
\text { de muestreo }\end{array}$ & $\begin{array}{c}\text { Área } \\
\text { (hectáreas) }\end{array}$ & $\begin{array}{c}\text { Número de sub-muestras de suelo } \\
\text { a extraer por área * }\end{array}$ \\
\hline Murtillar & 3,06 & 34 \\
\hline Sembradas & 3,60 & 36 \\
\hline Matorrales & 3,06 & 34 \\
\hline Matorrales & 3,18 & 35 \\
\hline Matorrales & 3,14 & 35 \\
\hline Matorrales & 3,36 & 37 \\
\hline Matorrales & 3,39 & 37 \\
\hline Matorrales & 3,31 & 36 \\
\hline Matorrales & 3,16 & 35 \\
\hline Coironal & 3,50 & 35 \\
\hline Coironal & 21,11 & 106 \\
\hline Coironal & 6,87 & 62 \\
\hline Coironal & 13,24 & 79 \\
\hline Coironal & 157,36 & 315 \\
\hline Vegas & 6,55 & 59 \\
\hline TOTAL & 237,89 & \\
\hline
\end{tabular}

*Los valores de la columna que indica el número de sub-muestras de suelo a extraer se encuentran aproximados al entero.

\section{CONCLUSIÓN}

La utilización de datos satelitales de libre disposición a través de softwares de código abierto como QGIS, podría reducir los costos de evaluación en los análisis desarrollados con percepción remota y SIG. Además, de acuerdo a los parámetros definidos en el presente estudio, permite identificar la superficie de áreas homogéneas según las clases de vegetación presentes en el predio y otras características topográficas.

La accesibilidad, asequibilidad y continuidad de los datos satelitales, y la creciente oferta de herramientas informáticas para el procesamiento y análisis de los datos, hacen que la incorporación de esta metodología para el muestreo de suelos en predios de grandes superficies, sea viable y de bajo costo. El uso de imágenes Landsat 8 son adecuadas para la identificación, cuantificación y distribución de las comunidades vegetales en predios de la región de Magallanes, obteniendo un índice de fiabilidad global de 85,6\% para el caso en estudio. No obstante, el uso de series temporales (disponibles), nuevas técnicas, algoritmos de clasificación supervisada de imágenes o imágenes de mayor resolución, pueden mejorar la calidad y precisión de los resultados.

El uso de MDE permite caracterizar el predio a partir de características morfométricas como la pendiente del terreno, no obstante, se requiere estudios locales que permitan conocer la precisión de ASTER GDEM.

Una vez definidas las áreas homogéneas, el muestreo debería ser selectivo de acuerdo a la comunidad a evaluar y el sitio que se quiere diagnosticar o intervenir, lo que ayudaría a disminuir los errores producto del muestreo, el tiempo de trabajo y facilitaría la logística en terreno.

\section{AGRADECIMIENTOS}

Parte del trabajo presentado en esta publicación fue apoyado por el Fondo de Fomento al Desarrollo Científico y Tecnológico (FONDEF D09I1036).

También se agradece las sugerencias del Dr. Dante Pinochet y del profesor Eduardo Mihovilovic.

\section{REFERENCIAS}

Athmania, D., Achour, H., 2014. External validation of the ASTER GDEM2, GMTED2010 and CGIAR-CSI-SRTM v4. 1 free access digital elevation models (DEMs) in Tunisia and Algeria. Remote Sensing 6(5), 4600-4620.

Bernier, R., 2000. Técnicas de muestreo de suelo para análisis de fertilidad, in: Bernier, R., (Ed.), Curso de capacitación para operadores del programa recuperación de suelos 
degradados INDAP décima región. Centro regional de investigaciones Remehue, Serie Actas $\mathrm{N}^{\circ}$ 02, Osorno, Chile, pp. 1-13.

Brady, N., Weil, R., 2008. The Nature and Properties of soils. Fourteenth Edition. New Jersey, USA.

Burrough, P.A., 1986. Principles of Geographic Information Systems for Land Resource Assessment. Monographs on Soil and Resources Survey No. 12. Oxford Science Publications, New York.

Burrough, P.A., McDonell, R.A., 1998. Principles of Geographical Information Systems. Oxford University Press, New York.

California Fertilizer Association, Soil Improvement Committee (CFASIC), 1995. Manual de Fertilizantes para Horticultura. Editorial UTHEA. $1^{\text {era }}$ Edición. Ciudad de México.

Chuvieco, E., 2006. Teledetección ambiental. Editorial Ariel Ciencia, Barcelona.

Collantes, M., Anchorena, J., Cingolani, A., 1999. The steppes of Tierra del Fuego: floristic and growth form patterns controlled by soil fertility and moisture. Plant Ecology $140,61-75$.

Congedo, L., Munafò, M., 2012. Development of a Methodology for Land Cover Classification in Dar es Salaam using Landsat Imagery. Sapienza University, Rome. http:// www.planning4adaptation.eu/Docs/papers/08_NWPDoM_for_LCC_in_Dar_using_Landsat_Imagery.pdf

Dinkins, C.P., Jones, C., 2008. Soil Sampling Strategies. Montana State University, Extension MT200803AG, pp. 1-4. http://store.msuextension.org/publications/AgandNaturalResources/MT200803AG.pdf

Domínguez, A., 1997. Tratado de fertilización. Tercera edición. Editorial Mundi Prensa. Madrid.

Enviromental Research Centre (ERC), 2004. Nutrient and trace element advice for grassland, tillage, vegetable and fruit crops. Teagasc, Johnstown Castle Research Centre, Wexford.

Food and Agriculture Organization of the United Nations (FAO), 1973. Guide to the calibration of soil tests for fertilizer recommendations. FAO Soils Bulletin, $N^{\circ} 18$. Roma.

Food and Agriculture Organization of the United Nations (FAO), 1986. Guía de fertilizantes y nutrición vegetal. Boletín FAO N ${ }^{\circ}$ 9. Roma.

Finck, A., 1988. Fertilizantes y fertilización. Editorial REVERTÉ, S.A. Barcelona.

Frame, J., Laidlaw, A.S., 2011. Improved grassland management. The Crowood Press, New edition. Wiltshire.

Foody, G.M., 2005. Local characterization of thematic classification accuracy through spatially constrained confusion matrices. International Journal of Remote Sensing 26(6), 1217-1228.

Gerding, V., Schlatter, J.E., 1996. Muestreo de suelos para evaluar su fertilidad, con énfasis en el régimen de elementos nutritivos. Facultad de Ciencias Forestales y Recursos Naturales, UACh, Valdivia, Chile.

Guerrero, A., 2000. El suelo, los abonos, y la fertilización de los cultivo. Mundi-Prensa. Madrid.

Hengl, T., Reuter, H., 2011. How accurate and usable is GDEM? A statistical assessment of GDEM using LiDAR data. Geomorphometry 2, 45-48.

Instituto Nacional de Estadísticas (INE), 2007. VII Censo
Agropecuario y Forestal. Resultados preliminares, 2006 - 2007. Gobierno de Chile, Santiago.

Ivelic-Sáez, J., Dörner, J., Radić, S., Valle, S., Dec, D., Zúñiga, F., 2014. Primeros alcances de calidad física de suelos de vega y coirón (Festuca gracillima) en Tierra del Fuego, Patagonia, Chile. Boletín $N^{\circ} 26$. Proceedings XII Congreso de la Sociedad Chilena de la Ciencia del Suelo. UMAG, Punta Arenas, Chile.

Johnson, B.A., Scheyvens, H., Shivakoti, B.R., 2014. An ensemble pansharpening approach for finer-scale mapping of sugarcane with Landsat 8 imagery. International Journal of Applied Earth Observation and Geoinformation 33, 218-225.

Knight, E., Kvaran, G., 2014. Landsat-8 Operational Land Imager design, characterization and performance. Remote Sensing 6, 10286-10305.

Knudby, A., Nordlund, L. M., Palmqvist, G., Wikström, K., Koliji, A., Lindborg, R., Gullström, M., 2014. Using multiple Landsat scenes in an ensemble classifier reduces classification error in a stable nearshore environment. International Journal of Applied Earth Observation and Geoinformation 28, 90-101.

León, R.J., Bran, D., Collantes, M., Paruelo, J.M., Soriano, A., 1998. Grandes unidades de vegetación de la Patagonia extra andina. Ecología Austral 8(2), 125-144.

Lopetegui, J., 2000. Evaluación de tres fuentes de fósforo de distinto grado de solubilidad, en una pradera permanente de la Décima Región. Tesis Licenciado en Ciencias Agrarias, Universidad Austral de Chile. 87 p.

Mallarino, A.P., Wittry, D.J., 2004. Efficacy of Grid and Zone Soil Sampling Approaches for Site-Specific Assessment of Phosphorus, Potassium, $\mathrm{pH}$, and Organic Matter. Precision Agriculture 5, 131-144.

Matus, F., 2006. Fertilidad de suelos y nutrición de cultivos; desarrollo de la experiencia en Chile, in: Luzio, W., Casanova, M. (Eds.), Avances en el conocimiento de los suelos de Chile. Maval Ltda. Santiago, pp. 193-239.

Maulik, U., Chakraborty, D., 2013. Learning with transductive SVM for semisupervised pixel classification of remote sensing imagery. ISPRS Journal of Photogrammetry and Remote Sensing 77, 66-78.

Miao, X., Patil, R., Heaton, J.S., Tracy, R.C., 2011. Detection and classification of invasive saltcedar through high spatial resolution airborne hyperspectral imagery. International Journal of Remote Sensing 32(8), 2131-2150.

National Plant Food Institute (NPFI), 2001. Manual de fertilizantes. Editorial Limusa, S.A. México.

Peng, G., Bing, W., Guangpo, G., Guangcan, Z., 2013. Spatial distribution of soil organic carbon and total nitrogen based on GIS and geostatistics in a small watershed in a hilly area of northern China. Plos one 8(12).

Phillips, R.D., Watson, L.T., Easterling, D.R., Wynne, R.H., 2014. An SMP soft classification algorithm for remote sensing. Computers \& Geosciences 68, 73-80.

Quantum GIS Development Team, 2015. Quantum GIS geographic information system. Open Source Geospatial Foundation Project. http://qgis.osgeo.org/ (acceso, 03.2015).

Radic, S., Fernández, A., Opazo, S., Mcadam, .J.H., Ivelic, J., 2013. Soil bulk density from grasslands in the Magallanes Region, Chile. $10^{\text {th }}$ International Conference of Agro- 
physics. Lublin, Poland.

Reuter, D.C., Richardson, C.M., Pellerano, F.A., Irons, J.R., Allen, R.G., Anderson, M., Thome, K.J., 2015. The Thermal Infrared Sensor (TIRS) on Landsat 8: Design overview and pre-launch characterization. Remote Sensing 7(1), 1135-1153.

Richards, J.A., 1999. Remote Sensing Digital Image Analysis, Springer-Verlag, Berlin.

Roberts, T.L., Henry, J.L., 2001. El muestreo de suelos: los beneficios de un buen trabajo. Potash and Phosphate Institute 42, 4-7.

Rodrigues, M.S., Corá, J.E., Fernandes, C., 2012. Soil sampling intensity and spatial distribution pattern of soils. Journal of the Brazilian Association of Agricultural Engineering 32(5), 852-865.

Rodríguez, J.M., 1991. Manual de fertilización. Alfa-Beta Impresores, Santiago de Chile.

Roy, D.P., Wulder, M.A., Loveland, T.R., Woodcock, C.E., Allen, R.G., Anderson, M.C., Zhu, Z., 2014. Landsat-8: Science and product vision for terrestrial global change research. Remote Sensing of Environment 145, 154-172.

Rozenstein, O., Karnieli, A., 2011. Comparison of methods for land-use classification incorporating remote sensing and GIS inputs. Applied Geography 31(2), 533-544.

Senf, C., Leitao, P.J., Pflugmacher, D., Van der Linden, S., Hostert, P., 2015. Mapping land cover in complex Mediterranean landscapes using Landsat: Improved classification accuracies from integrating multi-seasonal and synthetic imagery. Remote Sensing of Environment 156, 527-536.

Servicio Agrícola y Ganadero (SAG), 2003. El pastizal de Tierra del Fuego. Guía de uso, condición actual y propuesta de seguimiento para determinación de tendencia. Gobierno de Chile. Punta Arenas, XII Región de Magallanes y Antártica Chilena, Chile.

Servicio Agrícola y Ganadero (SAG), 2004a. El pastizal de Magallanes. Guía de uso, condición actual y propuesta de seguimiento para determinación de tendencia. Gobierno de Chile. Punta Arenas, XII Región de Magallanes y
Antártica Chilena, Chile.

Servicio Agrícola y Ganadero (SAG), 2004b. El pastizal de Ultima Esperanza y Navarino. Guía de uso, condición actual y propuesta de seguimiento para determinación de tendencia. Gobierno de Chile. Punta Arenas, XII Región de Magallanes y Antártica Chilena, Chile.

Steel, R., Torrie, J., 1980. Principles and Procedures of Statistics. A Biometrical Approach. Second Edition. Ed. McGraw-Hill, New York. USA.

Steiniger, S., Hunter, A.J., 2012. The 2012 free and open source GIS software map-A guide to facilitate research, development, and adoption. Computers, Environment and Urban Systems 39, 136-150.

Tejeda, H., Sadzawka, M.A. Araos, J., 2001. Análisis de Suelo, Foliar y de Agua para el Diagnóstico Nutricional y de Uso de Fertilizantes y Enmiendas, in: Román, S., (Ed.), Agenda del Salitre. $11^{\circ}$ Edición. Sociedad Química y Minera de Chile S.A. Santiago, pp. 197-230.

Thompson, A.N., Shaw, J.N., Mask, P.L., Touchton, J.T., Rickman D., 2004. Soil Sampling Techniques for Alabama, USA Grain Fields. Precision Agriculture 5(4), 345-358.

Turner, W., Rondinini, C., Petorelli, N., Mora, B., Leidner, A.K., Szantoi, Z., Woodcock, C., 2014. Free and open-access satellite data are key to biodiversity conservation. Biological Conservation 182, 173-176.

Wilson, J.P., 1999. Local, national, and global applications of GIS in agriculture, in: Longley, P.A., Maguire, D.J., Goodchild, M.F., Rhind, D.W., (Eds.), Geographical Information Systems: Volume 2, Management Issues and Applications. John Wiley and Sons, New York, pp. 981-989.

Yu, D., Zhang, Z., Yang, H., Shi, X., Tan, M., Sun, W., Wang H., 2011. Effect of soil sampling density on detected spatial variability of soil organic carbon in a red soil region of China. Pedosphere 21(2), 207-213.

Yonezawa, C., 2007. Maximum likelihood classification combined with spectral angle mapper algorithm for high resolution satellite imagery. International Journal of Remote Sensing 28(16), 3729-3737. 